\title{
Calidad de vida en pacientes operadas de bypass gástrico hace más de un año: Influencia del nivel socioeconómico
}

\author{
María José Leivala , Claudio Fuentealbaㄹ, Carolina Boggiano ${ }^{1}$, \\ Vivien G attás ${ }^{1}$, G ladys Barrera ${ }^{1}$, Laura Leiva ${ }^{1}$, \\ Daniel Bunout ${ }^{1}$, Sandra Hirsch ${ }^{1}$, M. Pía de la Maza ${ }^{1}$. \\ Q uality of life of patients subjected to \\ gastric bypass more than one year ago. \\ Influence of socioeconomic status
}

Background: The study of the effects of bariatic surgery on quality of life in patients of different socioeconomic levels (SEL) is worthwhile. Aim: To study quality of life (QoL), eating behaviour, depressive symptoms and sexuality in patients subjected to a gastric bypass (GBP) more than 1 year before. Material and methods: The sample was composed of 33 GPB patients (19 high SEL and 14 low SEL), and 27 non-operated women (18 high SEL and 9 low SEL) of similar weight and age, as controls. Assessment included medical history, anthropometry, radiological densitometry. Eating behaviour was assessed using the three factor eating questionnaire, quality of life using SF-36 and the Bariatric Analysis of Reporting Outcome System (BAROS) depressive symptoms were assessed using the Beck scale version II and sexual behaviour using the female sexual function index (FSFI). Results: QoL was lower in operated patients from low SEL, especially when compared to high SEL control women. Operated patients had a predominantly restrictive pattern of eating behavior. Eating behavior disorders were detected in 5 of 33 operated patients versus 4 of 27 controls $(p=n s)$. Sexual function was absent or dysfunctional in 22 operated versus 8 controls $(p=0,02)$. No significant differences were observed for depressive symptoms, between operated patients and controls. Conclusions: In the long term, QoL of bariatric patients, especially those from low SEL, is inferior to control women and Chilean general population. Operated patients have restrictive eating patterns and lower sexual satisfaction indexes. Frequency of depressive symptoms was high both in bariatric and control women (Rev Méd Chile 2009; 137: 625-33).

(Key words: Bariatric surgery; Quality of life; Socioeconomic factors)

Recibido el 6 de marzo, 2008. Aceptado el 27 de enero, 2009.

Financiado por Proyecto MULT 05/23-2, DI Universidad de Chile.

${ }^{1}$ Instituto de Nutrición y Tecnología de los Alimentos, Universidad de Chile, ${ }^{2}$ Departamento de Psiquiatría (Campus Sur), Facultad de Medicina, Universidad de Chile. Santiago de Chile. aPsicóloga Clínica

Correspondencia a: Dra. M Pía de la Maza. Macul 5540,

Santiago, Chile. E mail: mpmaza@inta.cl 
$\mathrm{E}^{1}$ impacto del bypass gástrico (BPG), sobre IMC y co-morbilidades médicas asociadas a la obesidad, es conocido. En cuanto a variables psicosociales, los estudios informan resultados a corto plazo, destacando mejorías significativas en la calidad de vida (CV) principalmente en factores tales como mayor movilidad y mejoría de las comorbilidades médicas ${ }^{1-3}$. Algunos estudios agrupan a pacientes con tiempos postoperatorios de tan amplio rango, que dificulta la comparación entre ellos ${ }^{4-6}$.

La CV se define como la percepción de un individuo de su posición en la vida, en el contexto cultural y el sistema de valores en que vive, en relación con sus metas, objetivos, expectativas, valores y preocupaciones ${ }^{7}$. La evaluación de la CV en un paciente representa el impacto que una enfermedad y su consecuente tratamiento tienen sobre la percepción del paciente de su bienestar ${ }^{8}$.

Existe asociación entre obesidad mórbida y comorbilidad psiquiátrica (mayor prevalencia de depresión, ansiedad, trastornos de la personalidad y trastornos de conducta alimentaria [TCA], específicamente el trastorno por atracones [TA]) y problemas en la esfera psicosocial ${ }^{9-12}$. Otros han detectado que la prevalencia de abuso sexual infantil es mayor entre obesos mórbidos operados de BPG, pero este factor no incidiría en la reducción de peso ${ }^{13}$.

Uno de los objetivos de la cirugía bariátrica es mejorar la deteriorada CV de los pacientes severamente obesos, por lo que diversos estudios han evaluado este parámetro. Los instrumentos más utilizados son el BAROS (Bariatric Analysis of Reporting Outcome System, diseñado específicamente para resultados de la cirugía bariátrica ${ }^{14-17}$ y el SF-36 (MOS 36-Item Short-Form Health Survey), cuestionario abreviado de CV relacionada a salud $^{18}$. Nguyen y cols utilizaron ambas escalas en pacientes con 3 meses de postoperatorio de BPG, confirmando que los puntajes de CV aumentaron considerablemente en relación al preoperatorio ${ }^{19}$. Utilizando 3 cuestionarios de $\mathrm{CV}$ en pacientes operados, Dymek y cols detectaron que efectivamente, el mayor impacto positivo en CV se observa en el primer semestre, estabilizándose posteriormente 20 .
Odgen y cols evaluaron los mecanismos psicológicos detrás del éxito en el proceso postquirúrgico, a través de diversas escalas. Observaron que los scores de CV, fatigabilidad, salud global y actividades de la vida diaria eran superiores en las pacientes operadas versus las no-operadas. Sin embargo, no detectaron diferencias en ansiedad, depresión y vigor entre ambos grupos ${ }^{21}$.

El objetivo del presente estudio fue evaluar la CV luego de más de 17 meses de postoperatorio, en mujeres chilenas. Se utilizó el SF-36 que ha sido validado en Chile para estudiar CV relacionada a enfermedad, y el BAROS, ampliamente usado en Chile. Para profundizar en aspectos psicológicos más específicos, se aplicaron tests de conducta alimentaria, depresión y sexualidad. Los resultados obtenidos fueron comparados con los de un grupo de mujeres con promedio de edad e IMC similar al de las pacientes operadas, pero no sometidas a cirugía ni tratamiento médico de la obesidad.

\section{PACIENTES Y MÉTODO}

Se incluyeron 33 pacientes que habían sido sometidas a BPG hacía más de 1 año, controladas por los autores o derivadas por nutriólogos de otros centros. Sus datos fueron comparados con los de 27 mujeres sanas, con edad e IMC comparables, pero que no habían consultado por problemas nutricionales, y eran conocidas por las pacientes o por los autores del estudio (grupo control). Ambos grupos fueron subdivididos en 2 categorías según nivel socioeconómico (NSE), en base a una encuesta de la unidad de Asistencia Social del Servicio de Salud Metropolitano Sur, de Santiago. Esta encuesta registra nivel educacional, tipo de trabajo, estabilidad laboral e ingreso mensual del grupo familiar. Según la clasificación del Instituto Consultor en Comencialización y Mercado (ICCOM*), se consideró NSE alto a los estratos $A B C 1$ y C2, y NSE bajo a C3, D y E.

En cada caso se efectuó una historia clínica, examen físico y exámenes de laboratorio clínico habitual (hemograma, glicemia, insulinemia, perfil lipídico, creatininemia, pruebas hepáticas, albúmi-

*www.iccom.cl 
na y TSH). Además se efectuaron mediciones antropométricas (peso, talla, circunferencia abdominal y de cadera) y densitometría de cuerpo entero en un equipo Lunar Prodigy.

Como pruebas psicométricas se aplicaron: BAROS, SF-36, escala de Beck II versión española de Páez para evaluar depresión ${ }^{22}$, Índice de Función Sexual Femenina (FSFI) ${ }^{23}$, Three Factor Eating Questionnaire (TFEQ) validado en Chile $^{24}$ y el Cuestionario de Patrones de Alimentación y Peso Revisado (QWEP-R) ${ }^{25}$.

Este trabajo fue aprobado por el Comité de ética del INTA, Universidad de Chile. Todas las pacientes y mujeres control firmaron un consentimiento informado antes de ser incluidas en el estudio.

Los promedios entre los 4 grupos estudiados fueron analizados a través de análisis de varianza y test post-hoc de Bonfermoni. Las proponciones entre grupos fueron analizadas a través de $\mathrm{Chi}^{2}$, y las asociaciones entre variables a través de comelación de Spearman, en el programa Stata 8.0 para Windows.

\section{RESUlTADos}

Las mujeres operadas tenían $45 \pm 9$ años de edad. El tiempo postoperatorio fluctuó entre 17 y 94 meses (mediana $=39$ meses). Las mujeres de NSE alto fueron operadas en el Hospital Clínico de la Pontificia Universidad Católica (8), Hospital Clínico de la Universidad de Chile (5), Clínica Indisa (3), Hospital Barros Luco-Trudeau (1), Clínica Dávila (1) y Hospital Militar (1). Las pacientes de NSE bajo fueron operadas en el Hospital Barros Luco-Trudeau (11) y Hospital Clínico de la Universidad de Chile (3). En promedio habían bajado $40,3 \pm 11 \mathrm{~kg}$, refiriendo haber manteniendo un peso relativamente estable en el último año.

El grupo control constaba de 27 mujeres de 41 \pm 9 años, 18 de NSE alto y 9 de NSE bajo, que no habían sido sometidas a ningún procedimiento bariátrico. Quince mujeres de este grupo tenían IMC normal y el resto sobrepeso u obesidad. Como criterio de inclusión adicional se solicitó que no estuviesen en tratamiento nutricional al momento del estudio.

En la Tabla 1 se presentan las variables antropométricas comparando pacientes y controles según NSE. Se aprecia que, si bien en promedio el IMC entre pacientes y controles era similar, al separar por NSE se observa que tanto controles como pacientes operadas de NSE alto tienen menor peso e IMC que sus pares de NSE bajo. Los exámenes de laboratorio habitual se

Tabla 1. Antropometría y composición corporal en pacientes operadas y controles de diferente nivel socioeconómico (N SE)

\begin{tabular}{|c|c|c|c|c|c|}
\hline & $\begin{array}{c}\text { Pacientes } \\
\text { operadas } \\
\text { N SE alto } \\
(n=19)\end{array}$ & $\begin{array}{c}\text { Pacientes } \\
\text { operadas } \\
\text { N SE bajo } \\
\text { (n =14) }\end{array}$ & $\begin{array}{l}\text { Mujeres } \\
\text { control } \\
\text { N SE alto } \\
(n=18)\end{array}$ & $\begin{array}{c}\text { Mujeres } \\
\text { control } \\
\text { N SE bajo } \\
\text { (n =9) }\end{array}$ & $\mathbf{p}$ \\
\hline $\begin{array}{l}\text { Talla (cm) } \\
0,422\end{array}$ & $161 \pm 5$ & $159 \pm 8$ & $161 \pm 8$ & $158 \pm 5$ & \\
\hline $\begin{array}{l}\text { Peso }(\mathrm{kg}) \\
0,0005\end{array}$ & $71 \pm 11$ & $82 \pm 10^{\mathrm{a} ß}$ & $64 \pm 10$ & $72 \pm 13$ & \\
\hline $\operatorname{IMC}\left(\mathrm{kg} / \mathrm{m}^{2}\right)$ & $27,4 \pm 4$ & $32,3 \pm 4^{\mathrm{a} ß}$ & $24,8 \pm 4$ & $29,2 \pm 6$ & 0,0001 \\
\hline Perímetro cintura $(\mathrm{cm})$ & $89 \pm 12$ & $99 \pm 11^{\mathrm{a}}$ & $83 \pm 10$ & $94 \pm 12$ & 0,0027 \\
\hline Porcentaje de grasa corporal (\%) & $39 \pm 6$ & $42 \pm 6^{\mathrm{a}}$ & $35 \pm 8$ & $42 \pm 5$ & 0,0098 \\
\hline
\end{tabular}

Valores corresponden a promedio \pm desviación estándar

$\%$ de grasa corporal calculado a través de DEXA

$\mathrm{p}=$ diferencias entre grupos analizada a través de ANOVA. Test post hoc de Bonferroni:

$\alpha=$ significativamente diferente de mujeres control de NSE alto.

$ß=$ significativamente diferente de pacientes operadas de NSE alto. 
encontraban dentro de rangos normales, excepto por 9 casos de anemia ferropénica entre las pacientes operadas versus 3 en el grupo control ( $p$ $=$ ns).

Los puntajes promedio obtenidos a través del SF-36 se encontraban entre 45 y 80 puntos en las pacientes y entre 54 y 90 puntos en el grupo control. Se detectó diferencias estadísticamente significativas en varios ítems de este test, específicamente entre las mujeres operadas de NSE bajo y las controles de NSE alto (Tabla 2). Esta tendencia se mantuvo al agrupar las variables por factor, predominando en los aspectos relacionados a salud mental. Según el BAROS, $87,8 \%$ de las pacientes operadas tendrían una CV sobre el rango de suficiente y sólo en 4 pacientes era insuficiente. Tanto el componente físico como el de salud mental del SF-36 se correlacionó negativamente con el puntaje del BAROS $(\mathrm{r}=-0,477 \mathrm{y}$ $0,483, \mathrm{p}=0,005$ y 0,004 , respectivamente).

En relación al ánimo, se observó una elevada frecuencia de puntajes altos para síntomas depresivos tanto en la muestra (12 de 33 pacientes) como en el grupo control ( 7 de 27 casos), sin

Tabla 2. Calidad de vida en pacientes operadas y controles de diferente nivel socioeconómico (N SE)

\begin{tabular}{|c|c|c|c|c|c|}
\hline $\begin{array}{l}\text { Variables } \\
\text { SF-36 } \\
\text { (puntaje máximo =100) }\end{array}$ & $\begin{array}{l}\text { Pacientes } \\
\text { O peradas } \\
\text { N SE alto } \\
\text { (n =19) }\end{array}$ & $\begin{array}{l}\text { Pacientes } \\
\text { O peradas } \\
\text { N SE bajo } \\
(n=14)\end{array}$ & $\begin{array}{l}\text { Mujeres } \\
\text { Control } \\
\text { N SE alto } \\
(n=18)\end{array}$ & $\begin{array}{l}\text { M ujeres } \\
\text { Control } \\
\text { N SE bajo } \\
(n=9)\end{array}$ & $\mathbf{p}$ \\
\hline \multicolumn{6}{|l|}{ Variables físicas } \\
\hline $\begin{array}{l}\text { Funcionamiento físico } \\
\text { Rol físico } \\
\text { Dolor corporal } \\
\text { Salud general } \\
\text { Cambios en salud } \\
\text { Componentes salud física }(\Sigma)\end{array}$ & $\begin{array}{c}80 \pm 18 \\
68 \pm 36 \\
70 \pm 20 \\
67 \pm 23 \\
62 \pm 32 \\
374,2 \pm 105\end{array}$ & $\begin{array}{c}69 \pm 25^{\alpha} \\
61 \pm 38 \\
53 \pm 32 \\
56 \pm 24^{\alpha} \\
55 \pm 34 \\
293,9 \pm 105\end{array}$ & $\begin{array}{c}91 \pm 10 \\
76 \pm 29 \\
76 \pm 22 \\
80 \pm 15 x \\
56 \pm 22 \\
378,9 \pm 69\end{array}$ & $\begin{array}{c}73 \pm 24 \\
81 \pm 35 \\
64 \pm 25 \\
54 \pm 26 \\
44 \pm 17 \\
315,9 \pm 89\end{array}$ & $\begin{array}{l}0,011 \\
0,407 \\
0,072 \\
0,006 \\
0,501 \\
0,075\end{array}$ \\
\hline Variables de salud & Mental & & & & \\
\hline $\begin{array}{l}\text { Salud mental } \\
\text { Rol emocional } \\
\text { Vitalidad } \\
\text { Función social } \\
\text { Componentes salud mental }(\Sigma)\end{array}$ & $\begin{array}{c}74 \pm 19 \\
60 \pm 40 \\
62 \pm 21 \\
73 \pm 24 \\
268,4 \pm 91\end{array}$ & $\begin{array}{c}46 \pm 25^{\alpha \beta} \\
50 \pm 41 \\
45 \pm 22^{\alpha} \\
47 \pm 32^{\alpha} \\
188,9 \pm 104^{\alpha}\end{array}$ & $\begin{array}{c}72 \pm 22 \\
633 \pm 9 \\
68 \pm 20 \\
82 \pm 21 \\
284,0 \pm 90\end{array}$ & $\begin{array}{c}67 \pm 19 \\
78 \pm 33 \\
58 \pm 17 \\
67 \pm 31 \\
269,9 \pm 74\end{array}$ & $\begin{array}{l}0,003 \\
0,426 \\
0,028 \\
0,006 \\
0,029\end{array}$ \\
\hline
\end{tabular}

Valores corresponden a promedio \pm desviación estándar. $\mathrm{p}=$ diferencias entre grupos analizadas con ANOVA Test post hoc de Bonferroni: $\alpha$ significativamente diferente de mujeres control de NSE alto.

$\beta$ significativamente diferente de pacientes operadas de NSE alto. $\chi$ significativamente diferente de mujeres control de NSE bajo.

Función física $\quad=$ Grado en que la salud limita las actividades físicas.

Rol físico $\quad=$ Grado en que la salud física interfiere en el trabajo y otras actividades dianias.

Dolor corporal = Intensidad del dolor y su efecto en el trabajo habitual, en el hogar o fuera de él.

Salud general = Valoración personal de la salud que incluye salud actual, perspectivas y resistencia a enfermar.

Cambios en salud $=$ Cambios observados en el último año.

Salud mental = Salud mental general, incluyendo depresión, ansiedad, control de la conducta o bienestar general.

Rol emocional = Grado en que los problemas emocionales interfieren en el trabajo o en otras actividades diarias.

Vitalidad $\quad=$ Sentimiento de energía y vitalidad versus sentimiento de cansancio y agotamiento.

Función social $\quad=$ Grado en que los problemas de salud física 0 emocional interfieren en la vida social habitual. 
alcanzar diferencias estadísticamente significativas (Tabla 3).

En cuanto a conducta alimentaria, evaluada a través del TFEQ, 88\% de las pacientes bariátricas, independiente del NSE, tenían un patrón de tipo restrictivo, estadísticamente diferente a las mujeres no operadas (Tabla 4). A través de la prueba QEWP-R, se observó que 5 de 33 pacientes de la muestra versus 4 de 27 mujeres del grupo control cabían dentro de una de las categorías de trastorno de conducta alimentaria TCA ( $p=0,371$ ) (Figura 1).

En cuanto a la sexualidad, la prueba FSFI reportó una función sexual dentro de los rangos de normalidad en 19 de las 27 mujeres del grupo control. En el grupo muestral en cambio, 11 de las 33 pacientes reportaron sexualidad normal, 6 pacientes clasificaron como disfunción sexual y 16 mujeres operadas refirió no tener actividad sexual alguna (Tabla 5).
DisCUSIÓN

El desafío de investigar acerca de CV en pacientes bariátricas, surgió en el contexto de la experiencia docente asistencial, en los Servicios de Cirugía y Psiquiatría del Hospital Barros Luco-Trudeau, Departamento de Psiquiatría Sur de la Facultad de Medicina e INTA, Universidad de Chile. Si bien los estudios de corto plazo indican que la mayor parte de la comorbilidad de la obesidad mórbida se corrige con la cirugía, incidiendo positivamente en CV, estos datos no coincidían plenamente con nuestras observaciones, en pacientes que asistieron a talleres grupales psicoeducativos durante la fase pre y postoperatoria. Por este motivo nos interesó tabular adecuadamente esta información, a través de un análisis transversal de pacientes operadas hace más de 2 años en promedio. Nos interesaba confrontar la información con la obtenida en mujeres no operadas $\mathrm{y}$, especialmente,

Tabla 3. Síntomas depresivos según escala de BECK, en pacientes y controles según nivel socioeconómico (N SE)

\begin{tabular}{|lcccc|}
\hline Escala de Beck & $\begin{array}{c}\text { Pacientes } \\
\text { operadas } \\
\text { N SE alto } \\
(\mathbf{n}=\mathbf{1 9 )}\end{array}$ & $\begin{array}{c}\text { Pacientes } \\
\text { operadas } \\
\text { N SE bajo } \\
(\mathbf{n}=\mathbf{1 4 )}\end{array}$ & $\begin{array}{c}\text { M ujeres } \\
\text { control } \\
\text { N SE alto } \\
(\mathbf{n}=\mathbf{1 8})\end{array}$ & $\begin{array}{c}\text { M ujeres } \\
\text { control } \\
\text { N SE bajo } \\
\text { (n =9) }\end{array}$ \\
\hline Normal (n) & 13 & 8 & 13 & 6 \\
$\begin{array}{l}\text { Depresión leve } \\
\text { Depresión moderada }\end{array}$ & 4 & 1 & 3 & 1 \\
$\begin{array}{l}\text { Depresión grave } \\
\text { Test de Chi }\end{array}$ & 2 & 4 & 1 & 2 \\
\hline
\end{tabular}

Tabla 4. Patrones de conducta alimentaria evaluada a través del TFEQ en pacientes operadas y controles

\begin{tabular}{|lcccc|}
\hline T FEQ & $\begin{array}{c}\text { Pacientes } \\
\text { operadas } \\
\text { N SE alto } \\
\text { (n =19) }\end{array}$ & $\begin{array}{c}\text { Pacientes } \\
\text { operadas } \\
\text { N SE bajo } \\
\text { (n =14) }\end{array}$ & $\begin{array}{c}\text { M ujeres } \\
\text { control } \\
\text { N SE alto } \\
\text { (n =18) }\end{array}$ & $\begin{array}{c}\text { M ujeres } \\
\text { control } \\
\text { N SE bajo } \\
\text { (n =9) }\end{array}$ \\
\hline Restricción & 15 & 14 & 8 & 5 \\
Desinhibición & 4 & 0 & 8 & 2 \\
Susceptibilidad al hambre & 0 & 0 & 2 & 2 \\
Test de chi2 & $\mathrm{p}=0,010$ & & & \\
\hline
\end{tabular}




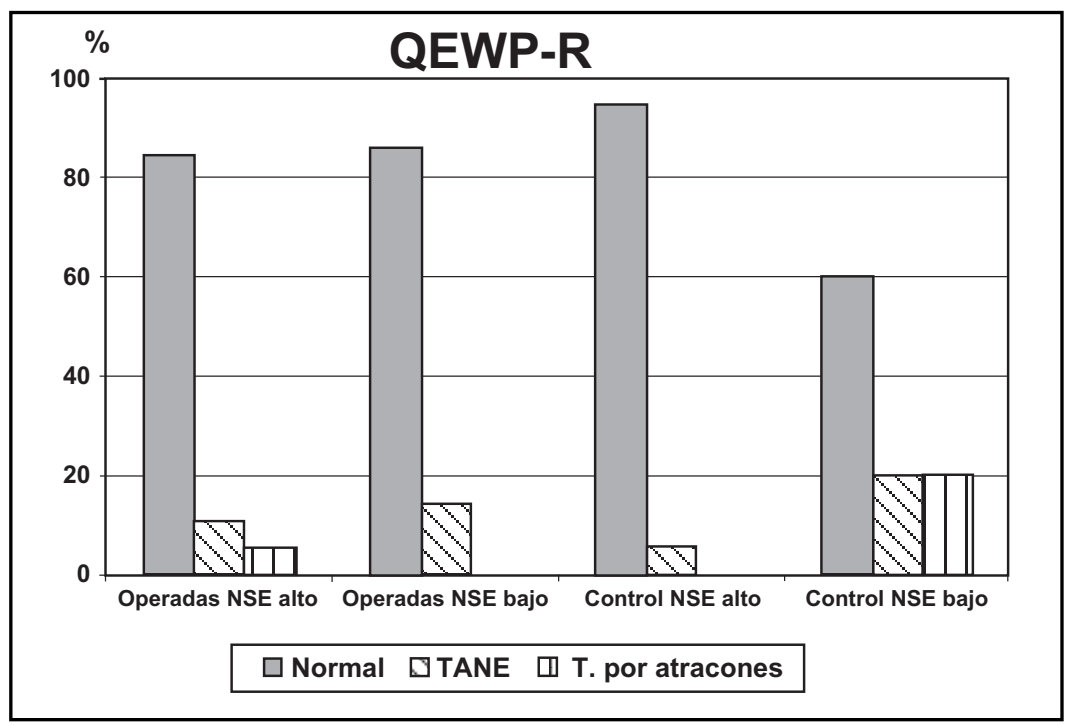

Figura 1. Frecuencia de trastornos de conducta alimentaria evaluada a través del QEWP-R en los 4 grupos estudiados. TANE = Trastorno no específico de la conducta alimentaria, $\mathrm{T}$. por ATRACONES = trastorno por atracones (según clasificación del DSM-IV R)

Tabla 5. Índice de función sexual femenina (FSFI) en pacientes operadas versus controles

\begin{tabular}{|lcccc|}
\hline $\begin{array}{l}\text { Resultado } \\
\text { FSFI }\end{array}$ & $\begin{array}{c}\text { Pacientes } \\
\text { operadas } \\
\text { N SE alto } \\
(\mathbf{n}=\mathbf{1 9 )}\end{array}$ & $\begin{array}{c}\text { Pacientes } \\
\text { operadas } \\
\text { N SE bajo } \\
(\mathbf{n}=\mathbf{1 4 )}\end{array}$ & $\begin{array}{c}\text { M ujeres } \\
\text { control } \\
\text { N SE alto } \\
\text { (n =18) }\end{array}$ & $\begin{array}{c}\text { M ujeres } \\
\text { control } \\
\text { N SE bajo } \\
\text { (n =9) }\end{array}$ \\
\hline $\begin{array}{l}\text { Sin actividad (n) } \\
\text { Normal (n) }\end{array}$ & 12 & 4 & 6 & 1 \\
$\begin{array}{l}\text { Disfunción (n) } \\
\text { Chi }\end{array}$ & 6 & 5 & 11 & 8 \\
\hline
\end{tabular}

que no hubiesen consultado por problemas nutricionales.

Los grupos estudiados resultaron relativamente homogéneos en cuanto a antropometría y laboratorio clínico, lo cual sugiere que la cirugía bariátrica lograría "acercar" a las pacientes operadas a la norma de la población general. Sin embargo, observamos que el patrón de conducta alimentaria era diferente en las pacientes operadas en relación al grupo control, por cuanto en las primeras predominaba un patrón de tipo restrictivo, lo que podría reflejar una capacidad de controlar conscientemente la ingesta alimentaria ${ }^{26-28}$. Este aspec- to es controversial: según la teoría de la restricción dietaria, esta conducta podría desencadenar atracones o sobrealimentación. En cambio, nuestra experiencia clínica coincide con otros estudios que demuestran que no es necesariamente una conducta de riesgo, sino que un tipo de relación más conciente y saludable con los alimentos, que permite lograr un balance energético adecuado para mantener el peso ${ }^{29}$. Por el contrario, las conductas de desinhibición y susceptibilidad al hambre sí serían predictoras de ganancia de peso $^{30}$. Así, en pacientes operados, la restricción podría contribuir a mantener el peso perdido ${ }^{31}$, 
en cambio la persistencia de atracones o de "picoteo" serían predictoras de aumento de peso post cirugía bariátrica ${ }^{32,33}$. Powers y cols señalan que la conducta de atracones se modifica producto de la cirugía, sin embargo, el síntoma vómito, relativamente frecuente en el postoperatorio, podría representar atracones fallidos ${ }^{34}$. En nuestras pacientes operadas, los vómitos disminuyeron con el tiempo, sólo 5 los presentaba al momento de la evaluación, y solamente en 2 casos la frecuencia era de 2 a 3 veces por semana (con lo cual podrían caer en la categoría de trastorno no específico de la conducta alimentaria (TANE). Por el contrario, llamó la atención el alto porcentaje de TCA (según criterios del DSM IV) en el grupo control, frecuencia que sería superior a la de la población general ${ }^{35}$ y acercándose más a las cifras de TCA entre obesos que consultan para bajar de peso $^{36,37}$. En nuestra experiencia, al realizar entrevistas clínicas semiestructuradas en pacientes en programa preparatorio para cirugía de bypass gástrico, $70 \%$ cumplió con los criterios para diagnosticar TCA en algún momento de su vida ${ }^{38}$, por lo tanto, a pesar de que las pacientes de esta muestra no presentaron mayor incidencia de TCA al compararlas con mujeres control, es un problema latente. Rusch y cols plantean ciertos criterios de riesgo para el desarrollo de TCA en el postoperatorio, tales como atracones, expectativas irreales con respecto a la cirugía, presión externa para bajar de peso, entre otros, y recomiendan controles periódicos para detectarlos, al menos durante los primeros 2 años de postoperatorio ${ }^{39}$.

Al comparar el nivel de CV entre las mujeres operadas versus controles, encontramos diferencias significativas, especialmente en los indicadores de salud mental, siendo más marcadas las diferencias entre el grupo control de NSE alto y el grupo de operadas de NSE bajo. Esta tendencia se repite para las pruebas específicas de ánimo y sexualidad. El BAROS en cambio clasificó a la mayoría de las pacientes con CV igual o superior a suficiente.

Nos parece importante destacar el ámbito de la sexualidad, dado que un alto porcentaje de las pacientes reportaron no tener actividad sexual, especialmente en el NSE alto. Resultaría interesante indagar más profundamente cuáles son las explicaciones a la base para esta conducta y cómo lo viven las pacientes. Una aproximación de tipo cualitativa podría darnos respuestas a esta interrogante.

En cuanto a la alta prevalencia de síntomas depresivos, tanto entre pacientes como controles, parece ser un hecho conocido en Chile. Según datos de la OMS, la prevalencia de depresión entre los chilenos es de 9,0\% para depresión mayor y de $8,0 \%$ para distimia ${ }^{40}$, siendo dos veces más frecuente en mujeres que hombres ${ }^{41}$.

Nuestros resultados permiten sugerir que, a pesar de una evidente mejoría en parámetros clínicos, la percepción de CV de las pacientes operadas no necesariamente mejora, tanto en los aspectos de salud física como de salud mental. Esto coincide con lo reportado por Odgen y $\operatorname{cols}^{21}$. Adicionalmente, al comparar con datos nacionales de CV obtenidos a través del SF-12 en el año $2006^{42}$, observamos que nuestras pacientes exhibieron puntajes menores a la mediana para el sexo femenino en todos los ítems. Nuestro estudio no permitió evaluar el cambio entre pre y post operatorio, para lograr una visión más global de la CV en este grupo de pacientes, sólo pudimos constatar el hecho de que la percepción de CV es deficiente a 2 ó 3 años de la cirugía. Nuestros resultados contrastan con los de otras investigaciones en el área ${ }^{19,20,43}$, que reportan mejorías significativas en CV después de la operación. Probablemente las diferencias se explican por el periodo en que fueron aplicados los tests, debido a que existe una diferente percepción a los 6 meses de postoperado que a los 2 a 3 años. Este aspecto podría relacionarse con la "percepción de cambio y transformación" al que hace referencia Bocchieri ${ }^{44}$, quien evidenció la sensación de un "antes y un después" de la operación. Esta transformación en una primera etapa tendería a ser definida como "totalmente positiva" para luego ir integrándose en sus aspectos positivos y negativos. Resulta relevante además que, al comparar por NSE, este deterioro es mayor en pacientes con NSE bajo, dato que podría relacionarse con el estudio mencionado ${ }^{44}$ en cuanto a las herramientas psicológicas disponibles en el paciente, las que determinarían el éxito, en términos de una adecuada adaptación a los cambios provocados por la cirugía. Podría concluirse que un mayor nivel social, económico y educacional facilitan el desarrollo de herramientas psicológicas para enfrentar situaciones de estrés vital, como sería la cirugía de bypass gástrico ${ }^{45}$. 
Serán necesarios estudios que complementen estos resultados y específicamente los aspectos de ánimo y sexualidad entre obesas mórbidas que han bajado de peso a través de la cirugía, utilizando metodología cualitativa. Esto es altamente relevante en vista de recientes estadísticas norteamericanas que informan disminución de mortalidad 7 años después del bypass gástrico, pero una mayor incidencia de accidentes y suicidios entre estos pacientes ${ }^{46}$.

\section{REFERENCIAS}

1. Papapietro K, Díaz E, Csendes A, Díaz JC, Braghetto I, BuRDILES P ET AL. [Effects of gastric bypass on weight, blood glucose, serum lipid levels and arterial blood pressure in obese patients]. Rev Méd Chile 2005; 133 : 511-6.

2. Goergen M, Arapis K, Limgba A, Schiltz M, Lens V, AzAgRa JS. Laparoscopic Roux-en-Y gastric bypass versus laparoscopic vertical banded gastroplasty: results of a 2-year follow-up study. Surg Endosc 2007; 21: 659-64.

3. Gould JC, Garren MJ, Boll V, Starling JR. Laparoscopic gastric bypass: risks vs benefits up to two years following surgery in super-super obese patients. Surgery 2006; 140: 524-9.

4. Sjostrom L, Lindroos AK, Peltonen M, Torgerson J, Bouchard C, Carlsson B et al. Swedish Obese Subjects Study Scientific Group Lifestyle, diabetes, and cardiovascular risk factors 10 years after bariatric surgery. N Engl J Med 2004; 351: 2683-93.

5. Pories WJ, Swanson MS, Macdonald KG, Long SB, MorRIS PG, BRown BM et al Who would have thought it? An operation proves to be the most effective therapy for adult-onset diabetes mellitus. Ann Surg 1995; 222: 339-50.

6. Christou NV, LoOK D, Maciean MD. Weight gain after short- and long-limb gastric bypass in patients followed for longer than 10 years. Ann Surg 2006; 244: 734-40.

7. WHOQOL GROUP. The world Health Organization quality of life assessment (WHOQOL) position paper from the World Health Organization. Soc Sci Med 1995; 41: 1403-9.

8. PAtrick D, ERicKson P. Health policy, quality of life: Health care evaluation and resource allocation. Oxford University Press New York 1993.

9. Glinski J, Wetzier S, Goodman E. The psychology of gastric bypass surgery. Obes Surg 2000; 11: 581-8.

10. SAUNDER R. Binge eating in gastric bypass patients before surgery. Obes Surg 1999; 9: 72-6.

11. Hsu L, SuLuvan S, BenotTi P. Eating disturbances and outcome of gastric bypass surgery: A pilot study. Int J Eat Disord 1997; 21: 385-90.
En conclusión, 2 a 3 años después del bypass gástrico se observó mantención del peso perdido a través de la cirugía y mejoría de los parámetros de laboratorio clínico, además de un patrón restrictivo en la conducta alimentaria, pero en cuanto a la CV, ésta sería de baja calidad, especialmente en los aspectos asociados a salud mental y específicamente en las áreas del ánimo y de la sexualidad. El NSE modula significativamente estas variables.

12. Biack D, Goldstein R, Mason E. Psychiatric diagnosis and weight loss following gastric surgery for obesity. Obes Surg 2003; 13: 746-51.

13. Grilo C, White M, Masheb R, Rothschild B, BurkeMartindale C. Relation of childhood sexual abuse and other forms of maltreatment to 12-month postoperative outcomes in extremely obese gastric bypass patients. Obes Surg 2006; 16: 454-60.

14. ORIA HE, MOOREHEAD MK. Bariatric analysis and reporting outcome system. Obes Surg 1998; 9: 94754.

15. Moorehead MK, Ardelt-Gattinger E, Lechner H, Oria HE. The Validation of the Moorehead-Ardelt Quality of Life Questionnaire II. Obes Surg 2003; 13: 684-92.

16. González JF, Gómez G, ARriagada G. Evaluación mediante score Baros de los resultados del bypass gástrico en el tratamiento de la obesidad mórbida. Rev Chil Cir 2006; 58: 365-70.

17. Awad W, Garay A, Martínez, Oñate V, Turu I, Yarmuch J. Descenso ponderal y calidad de vida mediante la cirugía de bypass gástrico con y sin anillo de calibración. Rev Chil Cir 2008; 60: 17-21.

18. Ware J, Snow K, Kosinski M. SF-36 Health Survey: Manual and Interpretation Guide. Lincoln, RI: Quality Metric; 1993.

19. Nguyen N, Varela E, Nguyen T, Wilson S. Quality of life assessment in the morbidly obese. Obes Surg 2006; 16: 531-3.

20. Dymek MP, Le Grange D, Neven K, Alverdy J. Quality of life after gastric bypass surgery: a cross-sectional study. Obes Res 2002; 10: 1135-42.

21. Odgen J, Clementi C, Aylwin S, Patel A. Exploring the impact of obesity surgery on patient's health status: a quantitative and qualitative study. Obes Surg 2005; 15: 266-72.

22. Beck AT, Steer RA, Ball R, Ranieri W. Comparison of Beck Depression Inventories -IA and -II in psychiatric outpatients. J Pers Assess 1996; 67: 588-97.

23. Rosen R, Brown C, Heiman J, Leiblum S, Meston C, Shabsigh R ET AL. The Female Sexual Function Index (FSFI): A multidimensional self-report instrument for the assessment of female sexual function. J Sex Marital Ther 2000; 26: 191-208. 
24. StunKard AJ, MesSick S. The three-factor eating questionnaire to measure dietary restraint, disinhibition and hunger. J Psychosom Res 1985; 29: 71-83.

25. FERguson KJ, Spitzer RL. Binge eating disorder in a community-based sample of successful and unsuccessful dieters. Int J Eat Disord 1995; 18: 167-72.

26. WeSTENHOEFER J. Dietary Restraint and Disinhibition: Is Restraint a Homogeneous Construct? Appetite 1991; 16: 45-55.

27. Kalarchian MA, Wilson GT, Broun RE, Bradiey L Effects of bariatric surgery on binge eating and related psychopathology. Eat Weight Disord 1999; 4: 1-5.

28. Bocchierr-Ricciardi LE, Chen EY, Munoz D, Fischer S, Dymer-Valentine M, Alverdy JC et al. Pre-surgery binge eating status: effect on eating behavior and weight outcome after gastric bypass. Obes Surg 2006; 16: 1198-204.

29. Wimamson DA, Martin CK, York-Crowe E, Anton SD, REDMAN LM, HAN H ET AL. Measurement of dietary restraint: validity tests of four questionnaires. Appetite 2007; 48: 183-92.

30. Blundell Je, Stubbs RJ, Golding C, Croden F, Alam R, WhyвRоW S ET AL. Resistance and susceptibility to weight gain: individual variability in response to a high-fat diet. Physiol Behav 2005; 86: 614-22.

31. SarLo-Lähteenkorva S, Rissanen A. Weight loss maintenance: determinants of long-term success. Eat Weight Disord 1998; 3: 131-5.

32. Hsu LK, Betancourt S, SuLvin SP. Eating disturbances before and after vertical banded gastroplasty: a pilot study. Int J Eat Disord 1996; 19: 23-34.

33. Burgmer R, Grigutsch K, Zipfel S, Wolf AM, De Zwaan M, HuSEMANN B ET AL. The influence of eating behavior and eating pathology on weight loss after gastric restriction operations. Obes Surg 2005; 15: 684-91.

34. Powers PS, Pérez A, Boyd F, Rosemurgy A. Eating pathology before and after bariatric surgery: a prospective study. Int J Eat Disord 1999; 25: 293300.

35. Feinstein SC, Sorosky A. Trastornos en la Alimentación (bulimia, obesidad, anorexia nervosa). Ed Nueva Visión. Buenos Aires, 1988.

36. Grucza RA, Przybeck TR, Cloninger CR. Prevalence and correlates of binge eating disorder in a community sample. Compr Psychiatry 2007; 48: 124-31.

37. De Zwaan M. Binge eating disorder and obesity. Int J Obes Relat Metab Disord 2001; 25: S51-5.

38. Fuentealba C, Leiva MJ. Trastornos de la conducta alimentaria y obesidad en Obesidad, un enfoque integral. Nestlé Chile 2007; 7: 97-110.

39. Rusch MD, ANDRis D. Maladaptive eating patterns after weight-loss surgery. Nutr Clin Pract 2007; 22: 41-9.

40. Minoletti A, Zaccaria A. Plan Nacional de Salud Mental en Chile: 10 años de experiencia. Rev Panam Salud Pública 2005; 18: 346-58.

41. Vicente B, Rioseco P, Saidivia S, Kohn R, Torres S. Estudio chileno de prevalencia de patología psiquiátrica (DSM-III-R/CIDI) (ECPP). Rev Méd Chile 2002; 130: 527-36.

42. http://epi.minsal.cl/epi/html/sdesalud/calidaddevida2006

43. Song AY, Rubin JP, Thomas V, Dudas JR, MarRa KG, FERNSTROM MH. Body image and quality of life in post massive weight loss body contouring patients. Obesity 2006; 14: 1626-36.

44. Bocchieri LE, Meana M, Fischer BL. Perceived psychosocial outcomes of gastric bypass surgery: a qualitative study. Obes Surg 2002; 12: 781-8.

45. Minayo MC, HaRTZ ZM, Buss P. Quality of life and health: a necessary debate. Ciencia y Saúde Colectiva 2000; ABRASCO 5: 7-18.

46. Adams TD, Gress RE, Smith SC, Halverson RC, Simper SC, ROSAMOND WD ET AL. Long-term mortality after gastric bypass surgery. N Engl J Med 2007; 357: 753-61. 